\title{
Telomerase expression shows differences across multiple regions of oligodendroglioma versus high grade astrocytomas but shows correlation with Mib-1 labelling
}

\author{
B K Kleinschmidt-DeMasters, T L Hashizumi, C-I Sze, K O Lillehei, A L Shroyer, \\ K R Shroyer
}

\begin{abstract}
Aims/Background-Telomerase is an enzyme that is expressed in most human neoplasms and is associated with tumour immortality. Determination of the point in neoplastic transformation at which telomerase is expressed may aid the understanding of tumour pathogenesis and progression. Despite numerous reports on telomerase, few studies have investigated its expression in high grade glial tumours. These studies, performed on archival banked, single brain tumour specimens, have shown conflicting results for oligodendrogliomas and unexpectedly negative results for telomerase expression in high grade astrocytomas, with one third to one half of glioblastoma multiformes being negative.
\end{abstract}

Methods-34 rapidly banked glioma specimens taken from patients undergoing gross total surgical resection of their tumours were studied. Telomerase expression was assessed across 3-8 sampled regions from each tumour by the telomeric repeat amplification protocol (TRAP) assay. Matched mirror image tissue samples were taken for histological analysis of tissue adequacy, statistical correlation of telomerase with tumour histological features, Mib-1 (a marker for cell cycling) labelling, and p53 immunohistochemistry. Results-All five well differentiated oligodendrogliomas were homogeneously telomerase negative and two of three untreated anaplastic oligodendrogliomas were homogeneously positive. In contrast, 10 of 14 high grade astrocytomas showed heterogeneity for telomerase expression across the multiple regions sampled. All glioblastoma multiformes and two of three anaplastic astrocytomas showed at least one region positive for telomerase. When test samples were individually assessed in both oligodendrogliomas and high grade astrocytomas, telomerase expression was associated with Mib-1 labelling $(p<0.001)$. For the entire group, telomerase expression was associated with grade of tumour, age of patient, and vascular endothelial proliferation (all p < 0.001).

Conclusions-This regional study clarifies that all glioblastoma multiformes are at least focally positive and that telomerase expression correlates with tumour grade in oligodendrogliomas. Homogeneity versus heterogeneity for telomerase expression across multiple regions of oligodendrogliomas versus high grade astrocytomas may provide important preclinical data on the use of antitelomerase agents in these adult glial tumours.

(f Clin Pathol 1998;51:284-293)

Keywords: telomerase; glioblastoma multiforme; oligodendroglioma; Mib-1

Telomerase is a ribonucleoprotein that is critical for the maintainence of continuous cell cycling, with down regulation resulting in cellular senescence. ${ }^{12}$ In tissues requiring continuous renewal, such as the gut, skin, ${ }^{34}$ and lymphoid tissues, ${ }^{5}$ telomerase continues to be expressed at low levels, while high telomerase expression is found almost exclusively in cells with unchecked proliferation and growthcancer cells. High telomerase levels are nearly always found in solid human tumours, ${ }^{6}$ although the grade and stage at which it is expressed for each given tumour type is variable. $^{7-11}$ Telomerase expression occurs in later phases of multistep tumour progression of some classes of tumours, ${ }^{12}$ and is especially characteristic of higher grade neoplasms. Information on telomerase expression in the human glial tumours, oligodendrogliomas and high grade astrocytomas (anaplastic astrocytomas and glioblastoma multiformes), has been limited and somewhat conflicting. ${ }^{13-15}$ Langford et al found that 19 of 19 oligodendrogliomas (grade not specified) were positive for telomerase, ${ }^{13}$ while our laboratory found negative telomerase expression in two of two low grade (grade 2 of 4; World Health Organisation (WHO) classification) oligodendrogliomas and positive telomerase expression in two of two anaplastic (grade 3 of 4) oligodendrogliomas (a pure oligodendroglioma and a mixed oligodendroglioma-anaplasticastrocytoma), suggesting that telomerase expression correlated with grade in oligodendrogliomas. ${ }^{14}$

However, both the studies of Langford and colleagues, ${ }^{13}$ our previous study, ${ }^{14}$ and a very recently published study by Nakatani and colleagues $^{15}$ agreed that a significant percentage of glioblastoma multiformes and anaplastic astrocytomas were negative for telomerase expression, a paradoxical finding in light of their high degree of malignancy and in contrast
Accepted for publication 9 December 1997 
to the almost uniform telomerase positivity of high grade systemic tumours. Using archivally banked, single tissue samples from each tumour, only 38 of 52 glioblastoma multiformes in the study of Langford and colleagues, ${ }^{13} 10$ of 19 glioblastoma multiformes in our previous study, ${ }^{14}$ and 12 of 20 glioblastoma multiformes in the study of Nakatani and colleagues ${ }^{15}$ were telomerase positive.

In this study, we used prospectively collected, gross total resection specimens obtained over the past 18 months from our institution to determine whether sampling of multiple regions throughout oligodendrogliomas and high grade astrocytomas, coupled with careful banking techniques and histological assessment of the adequacy of banked tissue, could clarify some of these conflicting and/or unexplained results. We hypothesized that, given the relative homogeneity versus heterogeneity of histological features in oligodendrogliomas versus high grade astrocytomas, such patterns might extend to telomerase expression across multiple regions in these tumours. We also sought to correlate telomerase expression with the pertinent histological features for each type of tumour, as well as with immunostaining for a cell cycle marker, Mib-1, using an antibody that stains a nuclear antigen which is expressed throughout active phases of the cell cycle (all phases except $\mathrm{G}_{0}$ ). In high grade astrocytomas (glioblastoma multiformes/anaplastic astrocytomas) we also correlated telomerase expression with protein immunohistochemistry for p53, via the well characterised D07 antibody. Few previous studies have undertaken detailed analyses of comparison between telomerase expression and Mib-1 or p53 expression, and virtually none have correlated histological features relevant to tumour type with telomerase.

\section{Materials and methods}

CRITERIA FOR PATIENT INCLUSION

Only patients who had undergone large, gross total resections of their neoplasms were eligible for the study. All but four patients were operated via stereotactic volumetric resection to achieve gross total resection. Because of concerns about accurate grading, glioma patients with single tissue samples or multiple stereotactic biopsies were not included in this study, even though this considerably limited the number of specimens available for analysis over the year and a half study period. Given the study design of obtaining multiple regional samples from each large resection specimen, these tissues represented uniquely banked specimens unavailable from national tumour tissue banks.

\section{PATIENT POPULATION}

Ten oligodendroglioma patients met the criteria for study. They ranged in age from 27 to 52 years and included eight women and two men. Eight of 10 patients had primary, untreated oligodendrogliomas. All five of the well differentiated oligodendrogliomas were untreated, whereas three of the five anaplastic oligodendrogliomas were untreated. The two anaplastic oligodendrogliomas were treated with both chemotherapy and cranial irradiation (table 1). All but one patient (oligodendroglioma case 9) are currently alive. Fourteen high grade astrocytoma patients met criteria for inclusion in the study, including 11 glioblastoma multiformes and three anaplastic astrocytomas. Patients ranged in age from 36 to 71 years, and included eight men and six women. Eight of 11 glioblastoma multiformes were untreated and

Table 1 Regional distribution of telomerase expression in oligodendrogliomas, and correlation with histological features and Mib-1 labelling

\begin{tabular}{|c|c|c|c|c|c|c|}
\hline $\begin{array}{l}\text { Regional specimen } \\
\text { numbers }\end{array}$ & $\begin{array}{l}\text { Patient age, sex and resection } \\
\text { date, tumour location }\end{array}$ & $\begin{array}{l}\text { Grade of neoplasm/ } \\
\text { treatment }\end{array}$ & $\begin{array}{l}T R A P \text { result of } \\
\text { regions }\end{array}$ & $\begin{array}{l}\text { Tumour necrosis of } \\
\text { regions }\end{array}$ & $\begin{array}{l}\text { Calcification listed } \\
\text { by region }\end{array}$ & $\begin{array}{l}\text { Microcysts listed } \\
\text { by region }\end{array}$ \\
\hline $\begin{array}{l}\text { Case } 1 \\
\# 152-156\end{array}$ & $\begin{array}{l}38 \mathrm{~F} ; 8 / 96 \\
\text { L. frontal }\end{array}$ & gr 2, ODG untreated & 5 of $5-v e$ & 5 of 5 absent & 5 of 5 absent & 5 of 5 absent \\
\hline $\begin{array}{l}\text { Case } 2 \\
\# 196-200\end{array}$ & $\begin{array}{l}35 \mathrm{~F} ; 11 / 96 \\
\text { L. frontal }\end{array}$ & gr 2, ODG untreated & 5 of $5-v e$ & 5 of 5 absent & 5 of 5 absent & $2+; 1+; 2+; 0 ; 0$ \\
\hline $\begin{array}{l}\text { Case } 3 \\
\# 235-239\end{array}$ & $\begin{array}{l}37 \mathrm{~F} ; 1 / 97 \\
\text { R. frontal }\end{array}$ & gr 2, ODG untreated & 5 of $5-v e$ & 5 of 5 absent & 5 of 5 absent & 5 of 5 absent \\
\hline $\begin{array}{l}\text { Case } 4 \\
\# 240-242\end{array}$ & $\begin{array}{l}29 \mathrm{~F} ; 1 / 97 \\
\text { R. frontal }\end{array}$ & gr 2, ODG untreated & 3 of $3-v e$ & 3 of 3 absent & 3 of 3 absent & $1+; 0 ; 0$ \\
\hline $\begin{array}{l}\text { Case } 5 \\
\# 243-248\end{array}$ & $\begin{array}{l}27 \mathrm{~F} ; 1 / 97 \\
\text { R. frontal }\end{array}$ & gr 2, ODG untreated & 6 of $6-v e$ & 6 of 6 absent & 6 of 6 absent & 6 of 6 absent \\
\hline $\begin{array}{l}\text { Case } 6 \\
\# 140-145\end{array}$ & $\begin{array}{l}50 \mathrm{~F} ; 5 / 96 \\
\text { R. frontal }\end{array}$ & gr 3, ODG untreated & 6 of 6 +ve & 6 of 6 absent & $2+; 2+; 2+; 1+; 0 ; 0$ & $\begin{array}{l}2+; 1+; 1+; 1+; \\
1+; 0\end{array}$ \\
\hline $\begin{array}{l}\text { Case } 7 \\
\# 216-223\end{array}$ & $\begin{array}{l}52 \mathrm{~F} ; 12 / 96 \\
\text { R. temporal }\end{array}$ & gr 3, ODG untreated & 8 of $8+v e$ & 8 of 8 absent & $\begin{array}{l}1+; 1+; 1+; 1+; 0 \\
2+; 2+; 2+\end{array}$ & 8 of 8 absent \\
\hline $\begin{array}{l}\text { Case } 8 \\
\# 210-215\end{array}$ & $\begin{array}{l}33 \mathrm{M} ; 10 / 96 \\
\text { L. frontal }\end{array}$ & gr 3, ODG untreated & $\begin{array}{l}1 \text { of } 6-\mathrm{ve} \\
5 \text { of } 6+\mathrm{ve}\end{array}$ & 6 of 6 absent & 6 of 6 absent & $\begin{array}{l}2+; 2+; 1+; 1+; \\
2+; 0\end{array}$ \\
\hline $\begin{array}{l}\text { Case } 9 \\
\# 177-180\end{array}$ & $\begin{array}{l}43 \mathrm{~F} ; 10 / 96 \\
\text { R. frontal }\end{array}$ & $\begin{array}{l}\text { gr 3, ODG-AA } \\
\text { recurrent }\end{array}$ & 4 of $4-v e$ & 4 of 4 absent & 4 of 4 absent & 4 of 4 absent \\
\hline $\begin{array}{l}\text { Case } 10 \\
\# 275-281\end{array}$ & $\begin{array}{l}35 \mathrm{M} ; 5 / 97 \\
\text { R. Parieto- } \\
\text { Temporal }\end{array}$ & gr 3, ODG recurrent & 7 of $7-v e$ & $\begin{array}{l}6 \text { of } 7 \text { absent } \\
1 \text { of } 7 \text { focal }\end{array}$ & $\begin{array}{l}0 ; 0 ; 2+; 0 ; 2+; 1+; \\
0\end{array}$ & 7 of 7 absent \\
\hline
\end{tabular}

All histological assessment performed on matched, mirror image tissue sections adjacent to tissue analysed for telomerase. All histological data are listed in order and correspond with regional telomerase specimen.

Scoring of high cell density: 0 , absent; $1+; 1 / 4$ of tumour; $2+, 1 / 4$ to $1 / 2$ of tumour; $3+,>1 / 2$ of tumour.

$\%$ necrosis is estimated from mirror image tissue stained with haematoxylin and eosin.

Nuclear anaplasia, nuclear enlargement, prominent nucleoli, and hyperchromatism; $0,1+, 2+$, semiquantitative estimate.

Microcysts, $0,1+, 2+, 3+$, semiquantitative estimate.

Mib-1, labelling index expressed as percentage, 1000 cells counted in highest labelled areas.

Scoring of lymphocytic inflammation on haematoxylin and eosin stained sections: 0 , none; $1+$, scattered lymphocytes in tumour or near vessels; $2+$, occasional perivascular lymphocytic cuffing; $3+$, prominent perivascular lymphocytic cuffing. 
all three anaplastic astrocytomas were untreated. The three treated glioblastoma multiformes received both chemotherapy and cranial irradiation (table 2). All but five patients (glioblastoma multiforme patients $1,2,4,5$, and 9) are currently alive.

\section{HANDLING OF TISSUE SPECIMENS}

All tumour tissue samples were obtained rapidly from resection specimens, within 15 minutes of surgical tissue removal. Tissue samples were taken from viable areas of tumour, avoiding areas of gross necrosis, especially in patients treated with radio- or chemotherapy. Three to seven anatomically separate areas of tumour tissue were sampled from each resection specimen, with the number of regional specimens taken from each case being determined by the volume of resected tissue available. Tissue was immediately snap frozen in a dry ice/ethanol mixture and stored at $-70^{\circ} \mathrm{C}$.

\section{GRADING OF TUMOURS}

Grading of tumours was performed on the formalin fixed, paraffin wax embedded sections, cut at $4 \mu \mathrm{m}$ and stained with hematoxylin and eosin. Both the adjacent tissue samples matched to the frozen tissue, as well as additional tissues submitted in toto from the resection specimens, were used for grading. Grading criteria for oligodendrogliomas divided the tumours into anaplastic versus well differentiated tumours and required the presence of increased mitotic activity and nuclear anaplasia in anaplastic variants (grade 3 of 4) and the absence of such features in well differentiated (grade 2 of 4 ) variants. In keeping with WHO criteria, ${ }^{16}$ grade 1 , on the four tiered scale, was not used for oligodendrogliomas. The presence of vascular endothelial prolifera- tion and/or necrosis was not required for an anaplastic tumour (grade 3). Each slide and section was assessed for the presence or absence of tumour necrosis, microcysts, gemistocytes, calcification, degree of cellularity, and nuclear anaplasia, with criteria as outlined in table 1. Nuclear anaplasia was defined as the presence of large, hyperchromatic nuclei with prominent nucleoli but not necessarily irregular, pleomorphic nuclear shape. All mitoses were counted by the same neuropathologist (BKD) to eliminate inter-observer variability. For grading and classification of anaplastic astrocytomas and glioblastoma multiformes, the WHO system was used, ${ }^{16}$ as modified by Burger and colleagues $^{17}$ and Nelson and colleagues. ${ }^{18}$ This defines glioblastoma multiformes as astrocytomas that possess cytological atypia, mitotic activity, usually vascular endothelial proliferation, and necrosis. By definition, anaplastic astrocytomas had cytological atypia and mitotic activity, but no necrosis. Histological features pertinent to high grade astrocytomas were assessed including necrosis, lymphocytic inflammation, gemistocytic features, and vascular endothelial proliferation. In addition, percentage of necrosis in each regional sample was recorded to clarify further whether extremely necrotic specimens might be more likely to show negative telomerase expression.

\section{Mib-1 ASSESSMENT}

Mib-1 assessment was performed on corresponding mirror image paraffin wax sections using the monoclonal Mib-1 antibody, $\alpha-\mathrm{Ki}-67$ (Immunotech Inc, Westbrook, Massachusetts, USA), and the indirect immunoperoxidase technique/avidin-biotin method following microwave treatment. In each case, 1000 cells were manually counted in the most heavily

Table 1 (continued)

\begin{tabular}{|c|c|c|c|c|c|c|}
\hline $\begin{array}{l}\text { Gemistocytes listed } \\
\text { by region }\end{array}$ & $\begin{array}{l}\text { Vascular endothelial } \\
\text { proliferation of region }\end{array}$ & $\begin{array}{l}\text { Cell density listed } \\
\text { by region }\end{array}$ & $\begin{array}{l}\text { Nuclear anaplasia } \\
\text { listed by region }\end{array}$ & $\begin{array}{l}\text { Mitoses listed } \\
\text { by region }\end{array}$ & $\begin{array}{l}\text { Mib-1 listed } \\
\text { by region }\end{array}$ & $\begin{array}{l}\text { Mean Mib-1 } \\
95 \% \text { CI }\end{array}$ \\
\hline 5 of 5 absent & 5 of 5 absent & $1+; 1+; 1+; 0 ; 0$ & $1+; 1+; 0 ; 1+; 0$ & $\begin{array}{l}3 ; 3 ; 1 ; 0 ; 0 \text { per } 20 \\
\text { HPF }\end{array}$ & $\begin{array}{l}10.9 ; 9.4 ; 5.1 ; 8.2 ; \\
9.0\end{array}$ & $\begin{array}{l}8.4 \\
6.2,10.6\end{array}$ \\
\hline 5 of 5 absent & 5 of 5 absent & $1+; 0 ; 1+; 0 ; 0$ & $1+; 0 ; 1+; 0 ; 0$ & $\begin{array}{l}9 ; 0 ; 2 ; 2 ; 0 \text { per } 20 \\
\mathrm{HPF}\end{array}$ & $3.8 ; 4.8 ; 4.1 ; 6.2 ; 5.4$ & $\begin{array}{l}4.9 \\
4.0,5.8\end{array}$ \\
\hline $1+; 0 ; 0 ; 0 ; 0$ & 5 of 5 absent & $0 ; 1+; 0 ; 0 ; 0$ & $0 ; 1+; 0 ; 0 ; 0$ & $\begin{array}{l}1 ; 0 ; 0 ; 0 ; 0 \text { per } 20 \\
\mathrm{HPF}\end{array}$ & $4.8 ; 5.2 ; 4.2 ; 4.2 ; 3.0$ & $\begin{array}{l}4.3 \\
3.6,5.0\end{array}$ \\
\hline 3 of 3 absent & 3 of 3 absent & $1+; 0 ; 0$ & $1+; 0 ; 0$ & $3 ; 0 ; 0$ per $20 \mathrm{HPF}$ & $1.6 ; 1.8 ; 1.8$ & $\begin{array}{l}1.7 \\
1.6,1.8\end{array}$ \\
\hline 6 of 6 absent & 6 of 6 absent & 6 of 6 absent & 6 of 6 absent & $\begin{array}{l}0 ; 0 ; 0 ; 0 ; 0 ; 0 \text { per } 20 \\
\text { HPF }\end{array}$ & $\begin{array}{l}1.4 ; 2.7 ; 2.2 ; 2.5 ; \\
0.3 ; 0.2\end{array}$ & $\begin{array}{l}1.6 \\
0.7,2.5\end{array}$ \\
\hline $1+; 0 ; 1+; 1+; 1+; 1+$ & $\begin{array}{l}5 \text { of } 6 \text { absent } \\
1 \text { of } 6 \text { focal }\end{array}$ & $1+; 3+; 3+; 3+; 3+; 3+$ & $\begin{array}{l}2+; 2+; 2+; 2+; \\
2+; 2+\end{array}$ & $\begin{array}{l}4 ; 3 ; 3 ; 4 ; 4 ; 3 \text { per } 20 \\
\text { HPF }\end{array}$ & $\begin{array}{l}10.9 ; 11.1 ; 7.9 ; 6.9 ; \\
21.2 ; \mathrm{ND}\end{array}$ & $\begin{array}{l}11.6 \\
7.0,16.2\end{array}$ \\
\hline $1+; 1+; 1+; 1+; 0 ; 1+; 0 ; 1+$ & $\begin{array}{l}6 \text { of } 8 \text { absent } \\
2 \text { of } 8 \text { focal }\end{array}$ & $\begin{array}{l}2+; 2+; 3+; 2+; 0 ; 3+ \\
3+; 2+\end{array}$ & $\begin{array}{l}2+, 2+, 2+; 2+ \\
1+; 2+; 2+; 2+\end{array}$ & $\begin{array}{l}3 ; 11 ; 9 ; 23 ; 7 ; 26 ; \\
22 ; 27 \text { per } 20 \mathrm{HPF}\end{array}$ & $\begin{array}{l}21.4 ; 19.6 ; 10.2 ; \\
17.8 ; 12.3 ; 19.4 \\
20.8 ; 29.2\end{array}$ & $\begin{array}{l}18.8 \\
14.5,23.1\end{array}$ \\
\hline $2+; 1+; 1+; 1+; 1+; 2+$ & 6 of 6 absent & $0 ; 1+; 1+; 2+; 2+; 1+$ & $\begin{array}{l}1+; 1+; 1+; 2+ \\
2+; 1+\end{array}$ & $\begin{array}{l}1 ; 2 ; 1 ; 5 ; 2 ; 1 \text { per } 20 \\
\text { HPF }\end{array}$ & $\begin{array}{l}3.6 ; 4.5 ; 3.8 ; 13.1 ; \\
7.3 ; 1.9\end{array}$ & $\begin{array}{l}5.7 \\
2.5,8.9\end{array}$ \\
\hline $0 ; 1+; 0 ; 0$ & 4 of 4 absent & 4 of 4 absent & $0 ; 0 ; 1+; 0$ & $\begin{array}{l}1 ; 1 ; 1 ; 0 \text { per } 20 \\
\text { HPF }\end{array}$ & $2.9 ; 5.5^{\star} ; 10.8^{\star} ; 6.0$ & $\begin{array}{l}6.3 \\
3.1,9.5\end{array}$ \\
\hline $1+; 2+; 2+; 1+; 1+; 2+; 0$ & $\begin{array}{l}3 \text { of } 7 \text { prominent } \\
3 \text { of } 7 \text { absent } \\
1 \text { of } 7 \text { focal }\end{array}$ & $0 ; 0 ; 0 ; 0 ; 1+; 0 ; 0$ & $\begin{array}{l}2+; 2+; 2+; 2+ \\
1+; 1+; 1+\end{array}$ & $\begin{array}{l}8 ; 1 ; 3 ; 1 ; 9 ; 1 ; 0 \text { per } \\
20 \mathrm{HPF}\end{array}$ & $\begin{array}{l}14.4 ; 9.7 ; 12.2 ; 13.6 \\
16.6 ; 8.6 ; 9.0\end{array}$ & $\begin{array}{l}12.0 \\
9.8,14.2\end{array}$ \\
\hline
\end{tabular}

Scoring of gemistocytic features on haematoxylin and eosin stained sections: $0,0-25 \%$ of cells in tumour section have gemistocytic features; $1+, 25-50 \%$ of cells in tumour section have gemistocytic features; $2+,>50 \%$ of cells in tumour section have gemistocytic features.

Scoring of vascular endothelial proliferation on haematoxylin and eosin stained sections: 0 , minimal to absent; $1+, 1-10$ proliferative vessels on section, usually focal; $2+, 10$ proliferative vessels on section, usually widespread.

Scoring of p53 immunohistochemistry: estimated percentage of labelled nuclei $(0,<1 \%, 1-10 \%, 10-30 \%, 30-60 \%,>60 \%)$.

*Insufficient tissue to count 1000 cells.

$\mathrm{R}$, right; L, left; ODG, oligodendroglioma; ODG-AA, mixed oligodendroglioma- anaplastic astrocytoma; GBM, glioblastoma multiforme (grade 4 of 4 astrocytoma, WHO classification); AA, anaplastic astrocytoma (grade 3 of 4 astrocytoma). HPF, high power ( $\times 400)$ microscopic field with a minimum of 20 HPF fields counted. $\mathrm{ND}$, not done because of inadequate amount of tumour on adjacent matched tissue. 
Table 2 Regional distribution of telomerase expression in high grade astrocytomas and correlation with histological features and Mib-1 and p53 labelling

\begin{tabular}{|c|c|c|c|c|c|}
\hline $\begin{array}{l}\text { Regional specimen } \\
\text { number }\end{array}$ & $\begin{array}{l}\text { Patient age, sex, resection date, } \\
\text { and tumour location }\end{array}$ & $\begin{array}{l}\text { Grade of neoplasm/ } \\
\text { treatment }\end{array}$ & $T R A P$ result of regions & $\begin{array}{l}\% \text { necrosis listed by } \\
\text { region }\end{array}$ & $\begin{array}{l}\text { Lymphocytic Inflammation } \\
\text { listed by region }\end{array}$ \\
\hline \multicolumn{6}{|c|}{ Glioblastoma multiforme } \\
\hline Case 1 & $68 \mathrm{M} ; 1 / 96$ & GBM untreated & 3 of $5+v e$ & \multirow[t]{2}{*}{$90 ; 30 ; 40 ; 40 ; 70$} & \multirow[t]{2}{*}{5 of $5-v e$} \\
\hline \# 135-139 & R. frontal & Died 11/96 & $\begin{array}{l}1 \text { of } 5-\mathrm{ve} \\
1 \text { of } 5+/-\end{array}$ & & \\
\hline Case 2 & $71 \mathrm{M} ; 2 / 96$ & GBM untreated & 1 of $5+$ ve & \multirow[t]{2}{*}{$50 ; 30 ; 50 ; 70 ; 50$} & \multirow{2}{*}{$3+; 2+; 3+; 3+; 2+$} \\
\hline \# 146-150 & R. parietal & Died 12/96 & $\begin{array}{l}3 \text { of } 5-\mathrm{ve} \\
1 \text { of } 5+/-\end{array}$ & & \\
\hline Case 3 & $68 \mathrm{M} ; 8 / 96$ & GBM untreated & 3 of $5+v e$ & \multirow[t]{2}{*}{$70 ; 0 ; 10 ; 80 ; 0$} & \multirow[t]{2}{*}{5 of $5-v e$} \\
\hline \# 157-161 & R. temporal & & 2 of $5-v e$ & & \\
\hline Case 4 & $34 \mathrm{M} ; 8 / 96$ & GBM; recurrent & 5 of $5+v e$ & \multirow[t]{2}{*}{5 of $5-v e$} & \multirow[t]{2}{*}{5 of $5-v e$} \\
\hline \# 162-166 & L. frontal & Died 11/96 & & & \\
\hline Case 5 & $50 \mathrm{~F} ; 9 / 96$ & GBM; recurrent & 4 of $5+v e$ & \multirow[t]{2}{*}{$40 ; 0 ; 15 ; 0 ; 5$} & 1 of $5+v e$ \\
\hline \# 167-171 & L. temporal & Died 11/96 & 1 of $5-\mathrm{ve}$ & & 4 of $5-v e$ \\
\hline Case 6 & $63 \mathrm{M} ; 10 / 96$ & GBM untreated & 2 of $5+v e$ & \multirow[t]{2}{*}{$10 ; 40 ; 70 ; 30 ; 5$} & 3 of $5+v e$ \\
\hline \# 172-176 & L. parieto-occipital & & $\begin{array}{l}1 \text { of } 5-\mathrm{ve} \\
2 \text { of } 5+/-\end{array}$ & & 2 of $5-v e$ \\
\hline $\begin{array}{l}\text { Case } 7 \\
\# 182-188\end{array}$ & $\begin{array}{l}59 \mathrm{M} ; 10 / 96 \\
\text { L. parieto-occipital }\end{array}$ & GBM untreated & $\begin{array}{l}6 \text { of } 7+\mathrm{ve} \\
1 \text { of } 7-\mathrm{ve}\end{array}$ & $70 ; 80 ; 70 ; 10 ; 0 ; 0 ; 90$ & 7 of $7-v e$ \\
\hline Case 8 & $41 \mathrm{~F} ; 12 / 96$ & GBM; recurrent & 3 of $3+v e$ & $0 ; 40 ; 30$ & 2 of $3+v e$ \\
\hline \# 206-208 & R. frontal & & & & 1 of $3-v e$ \\
\hline Case 9 & $69 \mathrm{M} ; 12 / 96$ & GBM untreated & 3 of $4+v e$ & $0 ; 0 ; 70 ;$ Scant tumour & 4 of $4-v e$ \\
\hline \# 224-227 & R. temporal & Died 2/97 & 1 of $4-v e$ & & \\
\hline $\begin{array}{l}\text { Case } 10 \\
\# 229-234\end{array}$ & $\begin{array}{l}67 \mathrm{~F} ; 12 / 96 \\
\text { L. temporal }\end{array}$ & $\begin{array}{l}\text { GBM with sarcomatous } \\
\text { areas, untreated }\end{array}$ & 6 of $6+v e$ & $0 ; 30 ; 0 ; 0 ; 0 ; 0$ & $0 ; 0 ; 1+; 1+; 2+; 0$ \\
\hline Case 11 & $41 \mathrm{~F} ; 5 / 97$ & GBM untreated & 2 of $3+v e$ & $5 ; 0 ; 0$ & 1 of $3+v e$ \\
\hline \# 284-286 & R. frontal & & 1 of $3-v e$ & & 2 of $3-v e$ \\
\hline \multicolumn{6}{|c|}{ Anaplastic astrocytomas } \\
\hline Case 12 & $36 \mathrm{M} ; 10 / 96$ & AA, untreated & 1 of $7+\mathrm{ve}$ & $0 ; 0 ; 0 ; 0$ & \multirow[t]{2}{*}{7 of $7-v e$} \\
\hline \# 189-195 & L. frontal & & $\begin{array}{l}5 \text { of } 7-v e \\
1 \text { of } 7+/-\end{array}$ & $0 ; 0 ; 0$ & \\
\hline $\begin{array}{l}\text { Case } 13 \\
\# 249-255\end{array}$ & $\begin{array}{l}53 \mathrm{~F} ; 1 / 97 \\
\text { R. parietal }\end{array}$ & AA, untreated & 4 of $7+$ ve 3 of $7-v e$ & $0 ; 0 ; 0 ; 0 ; 0 ; 0 ; 0$ & \multirow{3}{*}{$\begin{array}{l}2+; 2+; 1+ \\
2+; 1+; 1+; 1+ \\
0 ; 2+; 0 ; 0 ; 0 ; 0 ; 0\end{array}$} \\
\hline Case 14 & $48 \mathrm{~F} ; 3 / 97$ & AA, untreated & 7 of 7 -ve & $0 ; 0 ; 0 ; 0 ; 0 ; 0 ; 0$ & \\
\hline $263-269$ & L. tempero-parietal & & & & \\
\hline
\end{tabular}

For key see table 1.

$+/-$, negative telomerase expression at short $(<3$ day) exposure, positive at 7 day exposure.

labelled areas and positive nuclear labelling for labelled tumour nuclei was scored (without regard to staining intensity) as percentage of positive tumour nuclei. All actual Mib-1 scoring was performed by a single neuropathologist (BKD) to ensure standardisation of assessment and to reduce inter-observer variability, although confirmation of the general accuracy of the Mib-1 values was carried out independently by the second neuropathologist (CIS). Low Mib-1 labelling was considered to be $\leqslant 5.0 \%$, intermediate labelling index was defined as $5.1-10.9 \%$ and high labelling index was $\geqslant 11.0 \%$ positive cells. Confidence intervals of $95 \%$ were calculated for each case.

IMMUNOHISTOCHEMICAL LOCALISATION OF p 53 p53 immunohistochemistry used an anti-p53 antibody (D07, Biogenex, San Ramon, California, USA) which recognises both mutant and wild-type p53. The p53 immunohistochemistry was scored semi-quantitatively with estimates of $0 \%,<1 \%, 1-10 \%, 10-30 \%, 30-60 \%$, or $>60 \%$ ranges of positively labelled nuclei.

TELOMERIC REPEAT AMPLIFICATION PROTOCOL (TRAP ASSAY)

Frozen tissue blocks $(10-50 \mathrm{mg})$ were washed in $200 \mathrm{ml}$ ice cold wash buffer $(10 \mathrm{mM}$ Hepes$\mathrm{KOH}, \mathrm{pH} 7.5,1.5 \mathrm{mM} \mathrm{MgCl}, 10 \mathrm{mM} \mathrm{KCl}$, $1 \mathrm{mM}$ dithiothreitol), resuspended in 20$100 \mathrm{ml}$ ice cold lysis buffer $(10 \mathrm{mM}$ Tris- $\mathrm{HCl}$, pH 7.5, $1 \mathrm{mM} \mathrm{MgCl}_{2}, 1 \mathrm{mM}$ EGTA, $0.1 \mathrm{mM}$ phenylmethylsulfonyl fluoride, $5 \mathrm{mM}$ betamercaptoethanol, $0.5 \%$ CHAPS, $10 \%$ glycerol), and homogenised for five minutes using a disposable hand held homogeniser. The cellular suspension was incubated on ice for 30 minutes and then centrifuged for 30 minutes at $21000 \mathrm{~g}$ at $4^{\circ} \mathrm{C}$. The supernatant was removed and quick frozen in an ethanol/dry ice bath. The TRAP assay was performed as described by Kim and colleagues, ${ }^{6}$ and protein concentrations were standardised. ${ }^{19}$ As a telomerase positive control, an enzyme extract from either HEC-1A cells, an endometrial carcinoma cell line, or HeLa cells, a cervical epitheloid carcinoma cell line (both procured from American Type Culture Collection, Rockville, Maryland, USA), was included in each assay. Negative controls included RNase pretreatment of the tissue extracts, as well as a reagent control without tissue extracts. In addition, PCR inhibitors were ruled out by amplification of an internal 150 base pair (bp) control template. ${ }^{20}$ Electrophoresis of the TRAP assay products was performed on a $15 \%$ nondenaturing polyacrylamide gel at $50 \mathrm{~V} / \mathrm{cm}$. Following electrophoresis, the gels were dried with heat under vacuum and processed for autoradiography. Gels were exposed for 18-65 hours and again for seven days at $-70^{\circ} \mathrm{C}$. Positive results were defined as multiple consecutive (more than three) six nucleotide repeat bands for each lane, following seven days exposure. A single band, consistent with a size of $\sim 46 \mathrm{bp}$, was considered to be a primer-dimer artifact and was not considered positive.

SELECTION OF PATIENT GROUPS FOR TELOMERASE CORRELATION

Statistical association for the entire glioma study group was made for comparison of 
Table 2 continued

\begin{tabular}{|c|c|c|c|c|}
\hline $\begin{array}{l}\text { Gemistocytes listed } \\
\text { by region }\end{array}$ & $\begin{array}{l}\text { Vascular endothelial proliferation } \\
\text { listed by region }\end{array}$ & $\begin{array}{l}\% \text { p } 53 \text { immuno- histochemistry } \\
\text { listed by region }\end{array}$ & Mib-1 listed by region & $\begin{array}{l}\text { Mean Mib-1 } \\
95 \% \text { CI }\end{array}$ \\
\hline $0 ; 0 ; 0 ; 1+; 1+$ & $0 ; 2+; 1+; 1+; 1+$ & $0 ;<1 ;<1 ; 0 ;<1$ & $8.1 ; 13.1 ; 17.4 ; 30.5 ; 21.4$ & $\begin{array}{l}18.1 \\
10.6-25.6\end{array}$ \\
\hline $3+; 2+; 2+; 3+; 3+$ & 5 of $5-v e$ & 5 of $5-v e$ & $13.6 ; 6.3 ; 9.6 ; 6.1 ; 10.3$ & $\begin{array}{l}9.2 \\
6.5-11.9\end{array}$ \\
\hline 5 of $5-v e$ & 1 of $5+v e 4$ of $5-v e$ & $0 ; 0 ;<1 ;<1 ;<1$ & $7.1 ; 26.7 ; 16.8 ; 10.9 ; 24.8$ & $\begin{array}{l}17.3 \\
9.8-24.8\end{array}$ \\
\hline $0 ; 2+; 1+; 0 ; 2+$ & 1 of $5+v e 4$ of $5-v e$ & $<1 ;<1 ; 0 ; 0 ; 0$ & $31.9 ; 27.3 ; 28.0 ; 29.8 ; 15.3$ & $\begin{array}{l}26.5 \\
20.8-32.2\end{array}$ \\
\hline $\begin{array}{l}1 \text { of } 5+v e \\
4 \text { of } 5-v e\end{array}$ & $\begin{array}{l}3 \text { of } 5+v e \\
2 \text { of } 5-v e\end{array}$ & $0 ; 1-10 ; 10-30 ; 10-30 ; 30-60$ & $13.9 ; 7.4 ; 12.5 ; 15.2 ; 22.0$ & $\begin{array}{l}14.2 \\
9.6-18.8\end{array}$ \\
\hline $\begin{array}{l}4 \text { of } 5+v e \\
1 \text { of } 5-v e\end{array}$ & $2+; 2+; 1+; 2+; 2+$ & $<1 ;<1 ;<1 ; 1-10 ; 1-10$ & $34.9 ; 35.2 ; 28.5 ; 29.9 ; 34.7$ & $\begin{array}{l}32.6 \\
29.8-35.4\end{array}$ \\
\hline 7 of $7-v e$ & $\begin{array}{l}2 \text { of } 7+\mathrm{ve} \\
5 \text { of } 7-\mathrm{ve}\end{array}$ & $\begin{array}{l}1-10 ; 10-30 ; 1-10 ; 1-10 \\
10-30 ;<1 ;<1\end{array}$ & $29.6 ; 31.0 ; 21.3 ; 29.3 ; 21.4 ; 17.7 ; 20.9$ & $\begin{array}{l}24.5 \\
14.8-22.9\end{array}$ \\
\hline $0 ; 2+; 2+$ & 3 of $3+v e$ & $<1 ;<1 ; 1-10$ & $23.5 ; 30.2 ; 33.3$ & $\begin{array}{l}29.0 \\
23.3-34.7\end{array}$ \\
\hline $0 ; 0 ; 2+; 0$ & $2+; 0 ; 0 ; 0$ & $0 ; 0 ;<1 ; \mathrm{ND}$ & $32.0 ; 29.9 ; 22.1 ; \mathrm{ND}$ & $\begin{array}{l}28.0 \\
22.1-33.9\end{array}$ \\
\hline $\begin{array}{l}1 \text { of } 6+\mathrm{ve} \\
5 \text { of } 6-\mathrm{ve}\end{array}$ & 4 of $6+v e 2$ of 6 -ve & $\begin{array}{l}>60 ;>60 ;>60 ;>60 ; 30-60 \\
30-60\end{array}$ & $48.1 ; 28.2 ; 25.9 ; 37.5 ; 38.7 ; 35.7$ & $\begin{array}{l}35.7 \\
29.3-42.1\end{array}$ \\
\hline 3 of 3 neg & $1+; 0 ; 2+$ & $1-10 ; 1-10 ; 1-10$ & $26.9 ; 19.6 ; 11.4$ & $\begin{array}{l}19.3 \\
10.5-28.1\end{array}$ \\
\hline 7 of $7-v e$ & 7 of $7-v e$ & $\begin{array}{l}30-60 ;>60 ; 30-60 ; 10-30 ; 1-10 \\
30-60 ; 10-20\end{array}$ & $6.7 ; 6.6 ; 6.0 ; 7.2 ; 3.0 ; 6.0 ; 3.6$ & $\begin{array}{l}5.6 \\
4.4-6.8\end{array}$ \\
\hline $\begin{array}{l}0 ; 1+; 0 ; 1+ \\
1+; 2+; 2+\end{array}$ & 7 of $7-v e$ & $<1 ;<1 ; 0 ; 0 ;<1 ; 0 ; 0$ & $9.6 ; 13.4 ; 0.7 ; 2.4 ; 14.4 ; 3.0 ; 3.4$ & $\begin{array}{l}6.7 \\
2.5-10.9\end{array}$ \\
\hline 7 of 7 -ve & 7 of $7-\mathrm{ve}$ & $\begin{array}{l}30-60 ;>60 ;>60 ; 30-60 \\
30-60 ;>60 ;>60\end{array}$ & $9.3 ; 6.2 ; 2.7 ; 10.5 ; 2.6 ; 8.6 ; 17.1$ & $\begin{array}{l}8.1 \\
4.4-11.8\end{array}$ \\
\hline
\end{tabular}

Table 3 Statistical correlation of telomerase expression with: grade of tumour, Mib-1 labelling index, histological features, and 533 immunohistochemistry

\begin{tabular}{|c|c|c|}
\hline & Statistical test & $\begin{array}{l}\text { p value (excluding } \\
\text { \#145 \& 227) }\end{array}$ \\
\hline \multicolumn{3}{|l|}{ Entire glioma study group (untreated) } \\
\hline Grade of tumour & Fischer & $<0.001$ \\
\hline Mib-1 & General estimating equation & $<0.001$ \\
\hline Vascular endothelial proliferation & General estimating equation & $<0.001$ \\
\hline Age of patient & Logistic Regression & $<0.001$ \\
\hline \multicolumn{3}{|c|}{ Entire glioma study group (untreated + treated) } \\
\hline Grade of tumour & Fischer & $<0.001$ \\
\hline Mib-1 & General estimating equation & $<0.001$ \\
\hline Vascular endothelial proliferation & General estimating equation & 0.035 \\
\hline Age of Patient & Logistic Regression & $<0.001$ \\
\hline \multicolumn{3}{|l|}{ Oligodendrogliomas (untreated) } \\
\hline Grade of tumour & Fischer & $<0.001$ \\
\hline Mib-1 & General estimating equation & 0.097 \\
\hline Necrosis (present/absent) & General estimating equation & $<0.001$ \\
\hline Gemistocytic Predominance & General estimating equation & 0.103 \\
\hline Microcysts & General estimating equation & 0.376 \\
\hline tumour Cell Density & General estimating equation & 0.306 \\
\hline Mitoses & General estimating equation & 0.244 \\
\hline \multicolumn{3}{|c|}{ Oligodendrogliomas (untreated + treated) } \\
\hline Grade of tumour & Fischer & $<0.001$ \\
\hline Mib-1 & General estimating equation & 0.177 \\
\hline Vascular endothelial proliferation & General estimating equation & 0.944 \\
\hline Gemistocytic predominance & General estimating equation & 0.083 \\
\hline Microcysts & General estimating equation & 0.425 \\
\hline Tumour cell density & General estimating equation & 0.380 \\
\hline Mitoses & General estimating equation & 0.302 \\
\hline \multicolumn{3}{|c|}{ Glioblastoma multiforme/anaplastic astrocytomas (untreated) } \\
\hline Grade of tumour & Fischer & $<0.001$ \\
\hline Mib-1 & General estimating equation & $<0.001$ \\
\hline Degree of necrosis & General estimating equation & 0.192 \\
\hline Vascular endothelial proliferation & General estimating equation & $<0.001$ \\
\hline Gemistocytic predominance & General estimating equation & 0.001 \\
\hline Inflammation & General estimating equation & 0.007 \\
\hline p53 immunohistochemistry & General estimating equation & 0.545 \\
\hline \multicolumn{3}{|c|}{ Glioblastoma multiforme/anaplastic astrocytomas (untreated + treated) } \\
\hline Grade of tumour & Fischer & $<0.001$ \\
\hline Mib-1 & General estimating equation & $<0.001$ \\
\hline Degree of necrosis & General estimating equation & 0.437 \\
\hline Vascular endothelial proliferation & General estimating equation & 0.001 \\
\hline Gemistocytic predominance & General estimating equation & $<0.001$ \\
\hline Inflammation & General estimating equation & 0.002 \\
\hline p53 immunohistochemistry & General estimating equation & 0.319 \\
\hline
\end{tabular}




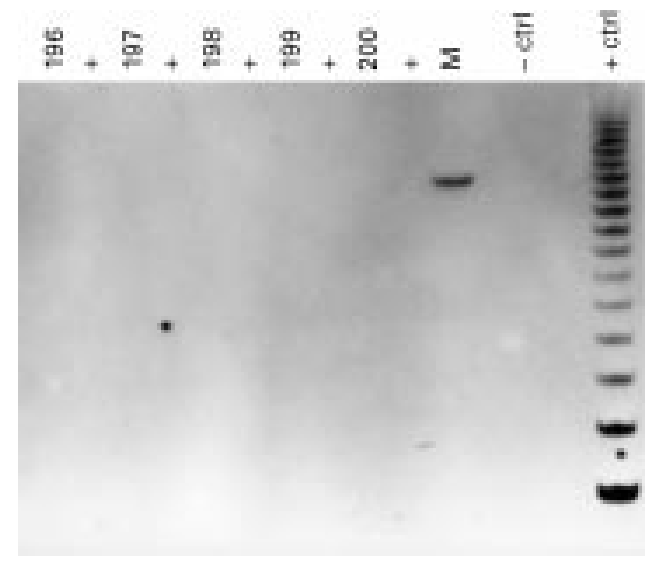

Figure 1 Detection of telomerase activity by the telomeric repeat amplification protocol (TRAP) assay in a well differentiated oligodendroglioma. Specimens 196-200 are five different regional tissue samples that do not demonstrate any bands (and therefore do not express telomerase) from case 2 (table 1). +, RNase pretreatment of specimen prior to PCR (RNase control); - Ctrl, reagent only negative control; + Ctrl, Hec-1A tissue lysate control.

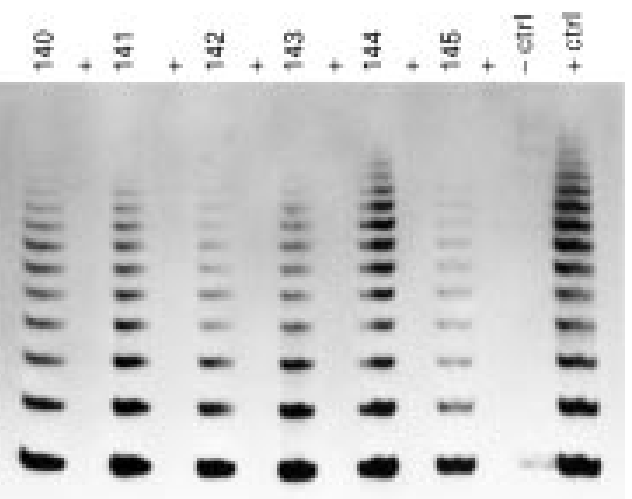

Figure 2 Detection of telomerase activity by the telomeric repeat amplification protocol (TRAP) assay in an anaplastic oligodendroglioma. Specimens 140-145 represent six different regional tissue samples from patient 6 (table 1) and displaying the characteristic multiband ladders diagnostic of positive telomerase expression.,$+ R N$ ase pretreatment of specimen prior to PCR (RNase control);

- Ctrl, reagent only negative control; + Ctrl, $\mathrm{Hec}-1 \mathrm{~A}$ tissue lysate control.

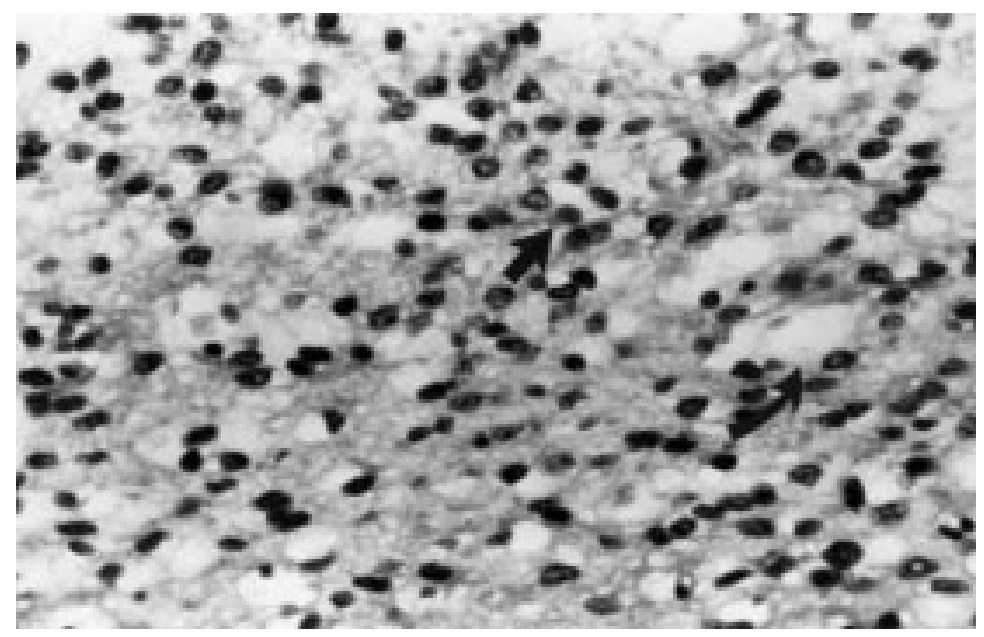

Figure 3 High power photomicrograph taken from the matched histological sample for one of the well differentiated oligodendrogliomas (oligodendroglioma case 2, specimen 199) illustrates the cellular monotony, perinuclear halos (straight arrow) and microcysts (curved arrow) typical of oligodendrogliomas, as well as bland nuclei with inconspicuous nucleoli seen in our well differentiated oligodendrogliomas group. Haematoxylin and eosin stained; $\times 350$ magnification. telomerase with grade of tumour, Mib-1 labelling index, vascular endothelial proliferation, and age of patient. For high grade astrocytomas and oligodendrogliomas, "untreated" patient groups within each tumour category were analysed separately from the "untreated + treated" group. This separation was based on our observation in treated oligodendrogliomas that the matched, adjacent tissue sections taken for histological analysis represented infiltrating edges of tumour that were hypocellular and possibly not representative of the tumour as a whole (see below). Our previous study had also noted that specimens from the infiltrating edges of gliomas might give falsely negative results. ${ }^{14}$ A similar observation of sample discordance in treated versus untreated high grade astrocytomas was not made; specifically, none of the treated high grade astrocytomas were sampled from the infiltrating edge as assessed on the matched histological specimen. However, groups were analysed separately in high grade astrocytomas to maintain uniformity of statistical analysis.

\section{STATISTICAL METHODOLOGY}

The statistical significance of telomerase activity relative to the determination of gender, treatment status, tumour type, and tumour histological diagnosis was determined using Fisher's exact test. ${ }^{21}{ }^{22}$ Association between telomerase expression, histological features, and Mib-1 labelling was undertaken using univariate analyses for patient and tumour summary data, as well as the generalized estimating equation for tumour specimen data. ${ }^{23}$ Statistical significance of Mib-1 variability was determined by the use of Bartlett's test for homogeneity of variance. ${ }^{24}$ Association between the Mib-1 labelling index and telomerase was also determined using the Wilcoxon rank sum test, a nonparametric equivalent to the two sample t-test. $^{25}$

\section{Results}

ENTIRE GLIOMA STUDY GROUP

Strong statistical relevance $(p<0.001$ for each) was found in both the "untreated" and the "untreated + treated" groups when correlating telomerase expression with grade of tumour, Mib-1 labelling index, or age of patient; moderate relevance was found when correlating telomerase expression with vascular endothelial proliferation in the "untreated + treated" group ( $\mathrm{p}=0.035)$, but strong statistical relevance was found when correlating telomerase expression with vascular dndothelial proliferation in the "treated" group $(\mathrm{p}<0.001)$ (table 3).

\section{OLIGODENDROGLIOMAS}

Telomerase activity was uniform across multiple regions of the oligodendrogliomas analysed, with all but one of the ten tumours (oligodendroglioma case 8) homogeneously positive or negative for expression (figs 1 and 2; table 1). Negative telomerase expression was seen in five well differentiated tumours with low cell density, little or no nuclear anaplasia, and low mitotic counts as assessed across mul- 


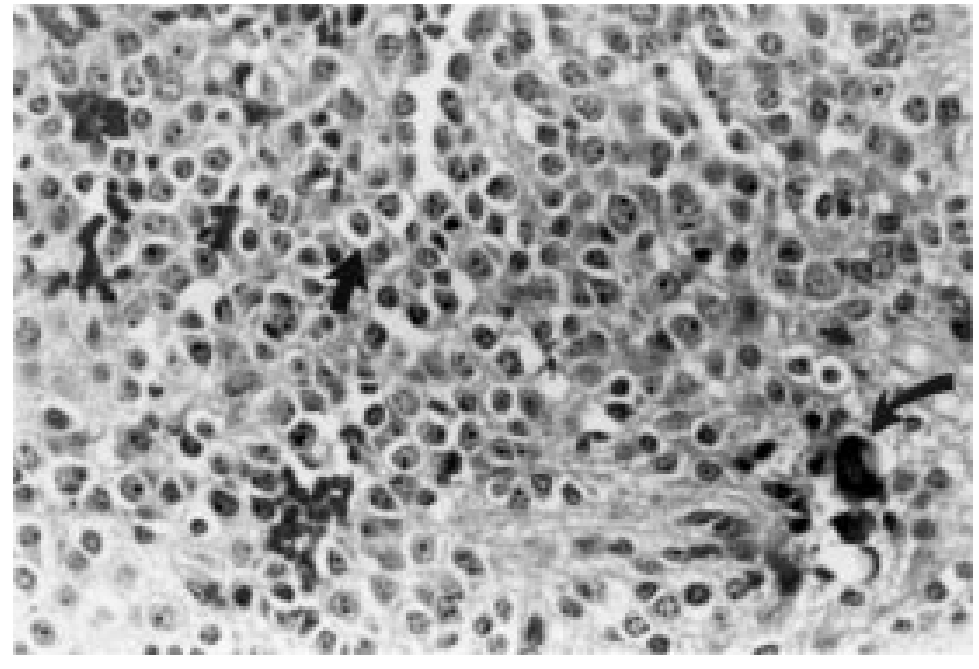

Figure 4 High power photomicrograph taken from the matched histological sample from one of the anaplastic oligodendrogliomas (oligodendroglioma case 6, specimen 144) illustrates the cellular monotony, perinuclear halos (straight arrow) and microcalcification (curved arrow) typical of oligodendrogliomas, as well as the more enlarged nuclei with prominent nucleoli seen in our anaplastic oligodendroglioma group. Haematoxylin and eosin stained; $\times 350$ magnification. tiple microscopic fields, as typified by case 2 (fig 3) (see table 1 for mitotic counts and Mib-1 labelling indices). The three untreated anaplastic oligodendrogliomas (cases 6-8) showed almost all regions with positive telomerase expression (fig 4). These tumours had Mib-1 labelling indices with wide variance, ranging from 1.9 to 29.2 , although all three untreated oligodendrogliomas had at least one area with a high Mib-1 labelling index $(\geqslant 11.0 \%$ ) (table 1$)$. Both treated anaplastic oligodendrogliomas (cases 9 and 10) showed that tumour was present in the corresponding adjacent mirror image tissue sections taken for tissue adequacy, but in small amounts, (especially oligodendroglioma case 9) and that the tissues were taken from the relatively less cellular, infiltrating edges of the tumour. Both treated anaplastic oligodendrogliomas were homogeneously negative for telomerase. No significant statistical relationship was found between Mib-1 and telomerase expression when either untreated or the whole oligodendroglioma cohort was analysed. However, when test samples were assessed individually, a strong association was found between Mib-1 and telomerase expression. No relationship was found between the presence or absence of microcysts, gemistocytes, or vascular endothelial proliferation and telomerase expression when either the entire oligodendroglioma group or the untreated cases only were analysed (table 3 ). Telomerase expression was noted in anaplastic oligodendrogliomas whether or not necrosis was absent or vascular proliferation was very focal or absent.

HIGH GRADE ASTROCYTOMAS

Of the 11 glioblastoma multiformes analysed, all showed at least one region positive for telomerase expression (table 2). However, only three of the patients (cases 4, 8, and 10) (table 2 ) showed homogeneously positive telomerase expression across all samples assessed. Weakly positive results, defined as three or more bands on the gel visible only at a seven day exposure (that is, $+/-$ results in table 2 ) or completely negative results were present in at least one region in eight of 11 glioblastoma multiformes studied (figs 5 and 6). Negative telomerase expression could not be accounted for by a high percentage of necrosis in the specimen, as assessed on the adjacent, mirror image specimen taken for histology. Only two of the three anaplastic astrocytomas showed definite positive telomerase expression in one of seven (case 12; table 2) and four of seven (case 13; table 2) regions studied. The third anaplastic astrocytomas (case 14; table 2) was completely negative for telomerase. Although the number of anaplastic astrocytomas in this study was small, an interesting association between the number of regions showing histological anaplasia and regions positive for telomerase expression was observed.

We analysed the glioblastoma multiformes/ anaplastic astrocytoma group separately for all patients versus untreated patients; however,

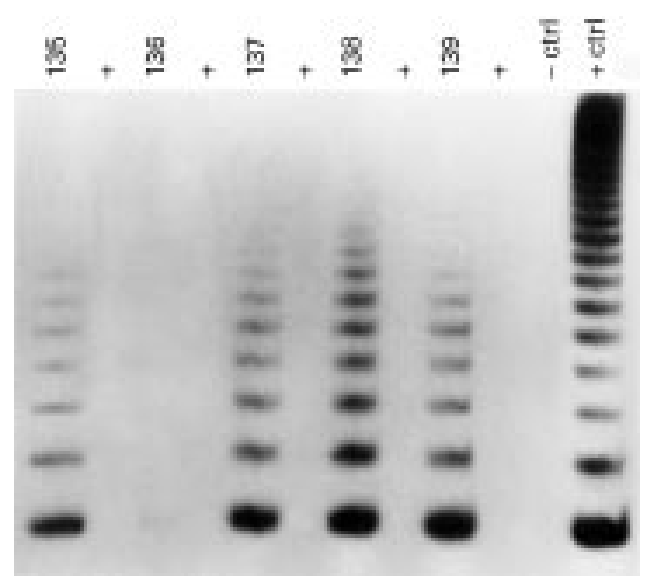

Figure 5 Detection of telomerase activity by the telomeric repeat amplification protocol (TRAP) assay in a glioblastoma multiforme from case 1 (table 2) with four positive regions (specimens 135, 137-139) and one negative region (specimen 136). + , RNase pretreatment of specimen prior to PCR (RNase control); - Ctrl, reagent only negative control; + Ctrl, Hec-1A tissue lysate control.

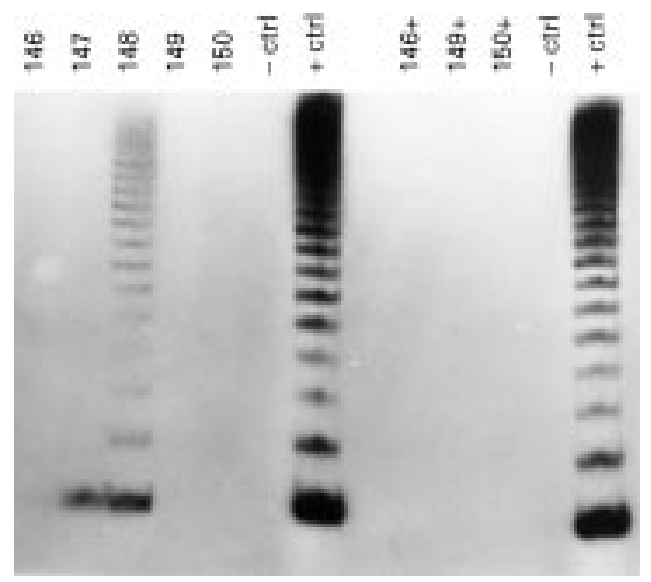

Figure 6. Detection of telomerase activity by the TRAP assay in a glioblastoma multiforme from case 2 (table 2) with four negative regions (specimens 146, 147, 149, and 150) and one positive region (specimen 148). The single band seen in lane 147 represents primer-dimer artifact. +, $R$ Nase pretreatment of specimen prior to PCR (RNase control); - Ctrl, reagent only negative control; + Ctrl, Hec-1A tissue lysate control. 
unlike the two treated oligodendroglioma cases, the tissue specimens from the treated glioblastoma multiformes patients were all predominately sampled in areas of high cell density and not the hypocellular, infiltrating edge of the neoplasm. As noted in table 2, previous treatment did not affect telomerase expression. A strong statistical association was found between Mib-1 and telomerase expression when either treated and untreated cases or untreated cases alone were analysed (table 3 ).

Decreased inflammation and positive telomerase expression showed significant correlation for both the high grade astrocytoma group as a whole and for the untreated cases (table 3). An inverse relation was also found between gemistocytic prominence and telomerase expression for both the whole glioblastoma multiformes/ anaplastic astrocytoma group and the untreated high grade astrocytoma group; the tumour with the most prominent lymphocytic inflammation and gemistocytic features also had one of the lowest mean Mib-1 labelling scores of the glioblastoma multiformes (case 2; table 2). A relation between vascular endothelial proliferation and telomerase expression for both the high grade astrocytoma group as a whole and for untreated patients only was noted. However, when this was adjusted for the fact that no anaplastic astrocytoma demonstrated vascular endothelial proliferation, no relation between telomerase and vascular endothelial proliferation remained (table 3 ).

REGIONAL DIFFERENCES IN p53

IMMUNOHISTOCHEMISTRY AND RELATION TO TELOMERASE EXPRESSION

Three of 11 glioblastoma multiformes showed regions with positivity in $10-30 \%$ of cells or more; there was slight regional variation for p53 immunoreactivity within each case (cases 5, 7, and 10; table 2). The strongest p53 immunoreactivity was noted in case 10 , the glioblastoma multiforme with sarcomatous transformation, and this case was homogeneously telomerase positive. However, two of the anaplastic astrocytomas (cases 12 and 14) also showed strong p53 immunoreactivity, but nearly opposite (mostly or completely negative) telomerase expression. Analysis between p53 immunoreactivity and telomerase expression did not reach statistical significance for either the untreated tumours or the glioblastoma multiformes/anaplastic astrocytoma group as a whole (table 3 ).

\section{Discussion}

The first objective of this study was to explain the paradoxical finding of the absence of telomerase expression in one half to one third of glioblastoma multiformes cases previously tested by TRAP analysis. ${ }^{13-15}$ Second, we were interested in whether increasing telomerase expression was associated with increasing grade of tumour, as we had previously suggested, ${ }^{14}$ and whether telomerase expression could be associated with Mib-1 labelling or histological features relevant to specific tumour type. Few previous studies have detailed correlation of relevant histological fea- tures with telomerase expression in tumours. The results of this study show that: (1) telomerase expression is found in all glioblastoma multiformes, albeit sometimes focally; (2) telomerase expression is associated with grade of tumour in both oligodendrogliomas $(p<0.001)$ and high grade astrocytomas (glioblastoma multiformes/anaplastic astrocytomas) ( $\mathrm{p}<0.001)$; and ( 3 ) telomerase expression is associated with Mib-1 labelling $(p<0.001)$, age of patient $(p<0.001)$, and vascular endothelial proliferation $(p=0.035)$ in the study group as a whole. Telomerase expression was more homogeneous throughout multiple regional samples in untreated oligodendrogliomas than in the high grade astrocytoma group, where only four of 14 glioblastoma multiformes/anaplastic astrocytomas showed homogeneity of telomerase expression in the multiple regional tumour samples $(p<0.001)$

Our original hypothesis for the first part of this study was that glioblastoma multiformes are highly heterogeneous tumours with protean gross and microscopic features and that this variability might extend to expression of tumour markers, including telomerase. This study demonstrates definitively that variations in telomerase expression exist from region to region within glioblastoma multiformes, suggesting that analysing only a single specimen from a glioblastoma multiformes might yield a nonrepresentative result. Our study explains, at least in part, the previously puzzling finding that the highest grade glial neoplasms, the glioblastoma multiformes, showed negative telomerase expression in one half to one third of cases studied, despite its nearly ubiquitous presence in other high grade neoplasms from other organ systems. ${ }^{13-15}$

A second objective of this study for high grade astrocytomas was to attempt to associate regional telomerase activity with common histological features found in these tumours, as well as with Mib-1 and p53 immunohistochemistry. No association between telomerase expression and the degree of necrosis in the tumour was noted. Specifically, a high percentage of necrosis in the tumour sample did not account for negative telomerase expression in this study, confirming our earlier findings. ${ }^{14} \mathrm{We}$ also examined lymphocytic inflammation in the tumour in relation to telomerase expression and found statistical association with decreased lymphocytic inflammation and increased telomerase expression. Although we originally hypothesised a possible relation between increased lymphocytic inflammation and positive telomerase expression in a tumour, because of the known positivity of non-neoplastic lymphocytes for telomerase (albeit low levels), ${ }^{26}$ this was not the case. The likely explanation for this statistically relevant finding is that greatly increased lymphocytic inflammation is common in gemistocytic astrocytic tumours that also show low Mib-1 labelling indices, ${ }^{27}$ with which telomerase levels inversely correlated.

Because at least low levels of telomerase can be found in non-neoplastic tissues that require constant renewal, ${ }^{29}$ and an association be- 
tween Mib-1 labelling and telomerase expression has been documented in other cancers, ${ }^{30}$ we investigated whether telomerase expression might be associated with Mib-1 labelling in gliomas. Strong statistical association could be seen between Mib-1 labelling and positive telomerase expression in this study for high grade astrocytomas. A recent study by Sallinen and colleagues $^{31}$ has also shown association between Mib-1 labelling and telomerase expression in astrocytomas, as well as heterogeneity of expression, using in situ hybridisation for telomerase RNA.

Our attempt to correlate p53 protein immunohistochemistry with telomerase expression in high grade astrocytomas was based on the known high frequency of p53 mutations in astrocytic tumours and the suggestion that clonal expansion of $\mathrm{p} 53$ mutated cells occurs in the malignant progression of astrocytomas, ${ }^{32}$ although Louis has suggested that $\mathrm{p} 53$ protein accumulation can occur in high grade astrocytomas for reasons other than p53 mutation. ${ }^{33}$ We found no relation between telomerase expression and p53 protein immunohistochemistry in high grade astrocytomas in this study. The application of hTR in situ hybridisation will make it possible to readdress the question of $\mathrm{p} 53$ protein accumulation and telomerase expression at the individual cell level.

A third objective of this study was the investigation of whether telomerase expression could be associated with grade of tumour, histological features, and Mib-1 labelling in oligodendrogliomas. In general, low grade oligodendrogliomas were telomerase negative and untreated anaplastic oligodendrogliomas were telomerase positive, confirming our initial findings that telomerase expression is associated with tumour grade. ${ }^{14}$ This finding may contrast with the original study by Langford $e t$ al, who found 19 of 19 oligodendrogliomas (grade not designated) positive. ${ }^{13} \mathrm{~A}$ strong statistical association was noted between grade of oligodendroglioma, as defined in our current study, and telomerase expression.

Although most neuropathologists divide oligodendrogliomas into well differentiated and anaplastic variants, the criteria for grading oligodendrogliomas are less precise than those used for high grade astrocytomas. Hence, we analysed a greater number of potentially relevant ${ }^{34-37}$ histological features in oligodendrogliomas than in high grade astrocytomas, and examined this correlation with telomerase expression. Our study found no association between telomerase expression and most of these debated histological features in the untreated oligodendrogliomas that we analysed, including microcyst formation, high cell density, gemistocytes, or mitoses (table 3). Only correlation between necrosis and telomerase expression in this group reached statistical significance.

We were also interested in associating telomerase with Mib-1 labelling in oligodendrogliomas because several investigators ${ }^{38}{ }^{39}$ had concluded that the Mib-1 labelling index has prognostic significance for oligodendrogliomas independent of other prognostic indicators such as patient age, site of tumour, and grade. However, Schiffer and colleagues ${ }^{37}$ noted that although a shorter survival was found for oligodendrogliomas with Mib-1 labelling indices of greater than $15 \%$, there was a large overlap between the Mib-1 labelling index ranges for "benign" and "malignant" oligodendrogliomas. They concluded that Mib-1 analysis was a useful prognostic tool, but not of absolute reliability in individual cases, a conclusion which we agree with. In our current study, when within patient variability was accounted for, no association was found between Mib-1 labelling and telomerase expression when either untreated oligodendrogliomas alone or the whole oligodendrogliomas group was analysed. However, when test samples were assessed individually, a strong association was found between the Mib-1 labelling index and telomerase expression. From this study, telomerase expression in oligodendrogliomas appears to be at least as useful as Mib-1 labelling or histological assessment in defining two groups of oligodendrogliomas.

In conclusion, this study has shown that: (1) telomerase expression is strongly associated with grade of tumour in both oligodendrogliomas and high grade astrocytomas; (2) telomerase expression is strongly associated with Mib-1 labelling, age of patient, grade of tumour, and vascular endothelial proliferation in the glioma study group as a whole; and (3) all glioblastoma multiformes tested to date are, in fact, at least focally telomerase positive. Future studies will investigate the usefulness telomerase expression as an adjunct to the grading of oligodendrogliomas.

Dr A L Shroyer was funded and sponsored, in part, by the Department of Veterans Affairs Office of Performance and Program for Cooperative Studies. The authors gratefully acknowlHoernig and Ms V McHenry; the manuscript preparation of Ms N Hart, Ms V Parker, Ms G Woodward, and Ms L C Evans; the photographic expertise of Mr B McCullough; and the patient follow up data gathering of Ms D Hopper, and Mrs A Ebert.

1 Axelrod N. Of telomeres and tumors. The enzyme, telomerase, may be switched on in tumor cells. Inhibitors of this ase, may be switched on in tumor cells. Inhibitors of this enzyme might constitute

2 Greider CW, Blackburn EH. Telomeres, telomerase and cancer. Sci Am 1996;274:92-7.

3 Ramirez RD, Wright WE, Shay JW, et al. Telomerase activity concentrates in the mitotically active segments of human hair follicles. F Invest Dermatol 1997;108:113-17.

4 Taylor RS, Ramirez RD, Ogoshi M, et al. Detection of telomerase activity in malignant and nonmalignant skin conditions. F Invest Dermatol 1996;106:759-65.

5 Yamada O, Motoji T, Mizoguchi H. Up-regulation of telomerase activity in human lymphocytes. Biochim Biophys Acta 1996;1314:260-6.

6 Kim NW, Piatyszek MA, Prowse KR, et al. Specific association of human telomerase activity with immortal cells and cancer. Science 1994;266:2011-15.

7 Broccoli D, Young, JW, de Lange T. Telomerase activity in Broccoli D, Young, JW, de Lange T. Telomerase activity in Sci USA 1995;92:9082-6.

8 Chadeneau C, Hay K, Hirte HW, et al. Telomerase activity associated with acquisition of malignancy in human colorectal cancer. Cancer Res 1995;55:2533-6.

9 Hiyama E, Gollahon L, Kataoka T, et al. Telomerase activity n human breast tumors. F Natl Cancer Inst 1996;88:11622.

10 Hiyama K, Hiyama E, Ishioka S, et al. Telomerase activity in small-cell and non-small-cell lung cancers. 7 Natl Cancer Inst 1995;87:895-902.

11 Hiyama E, Yokoyama $\mathrm{T}$, Tatsumoto $\mathrm{N}$, et al. Telomerase activity in gastric cancer. Cancer Res 1995;55:3258-62.

12 Blasco MA, Rizen M, Greider CW, et al. Differential regulation of telomerase activity and telomerase RNA during tion of telomerase activity and telomerase RNA diti-stage tumorigenesis. Nat Genet 1996;12:200-4.

13 Langford LA, Piatyszek MA, Xu R, et al. Telomerase activity in human brain tumors. Lancet 1995;346:1267-8. 
14 DeMasters BKK, Markham N, Lillehei KO, et al. Differential telomerase expression in human primary intracranial tial telomerase expression in human primary
tumors. Am $\mathcal{F}$ Clin Pathol 1997;107:548-54.

15 Nakatani K, Yoshimi N, Mori H, et al. The significant role of telomerase activity in human brain tumors. Cancer 1997;80:471-6.

16 Kleihues P, Burger PC, Scheithauer BW. Histologic typing of tumors of the central nervous system, 2nd ed. World Health Organisation. Berlin: Springer Verlag, 1993.

17 Burger PC, Vogel FS, Green SB, et al. Glioblastoma multiforme and anaplastic astrocytoma: pathologic criteria and prognostic implications. Cancer 1985;56:1106-11.

18 Nelson JS, Tsukada Y, Schoenfeld D, et al. Necrosis as a prognostic criterion in malignant supratentorial astrocytic gliomas. Cancer 1983;52:550-4.

19 Bradford MM. A rapid and sensitive method for the quantitation of microgram quantities of protein utilizing the principle of protein-dye binding. Anal Biochem 1976;72:248-54.

20 Wright WE, Shay JW, Piatyszek MA. Modifications of a telomeric repeat amplification protocol (TRAP) result in telomeric repeat amplification protocol (TRAP) result in Res 1995;23:3794-5.

21 Rosner BA. Hypothesis testing: categorical data. In: Fundamentals of biostatistics. Boston: Duxbury Press, 1982:22553, 308-17.

22 Mehta CR, Patel NR. A network algorithm for performing Fisher's exact test in $\mathrm{r} x \mathrm{c}$ contingency tables. $\mathcal{F} \mathrm{Am}$ Stat Assoc 1983;78:427-34

23 Nelder JA, Wedderbum, RWM. Generalized linear models. f Roy Stat Soc 1972;135:370-84

24 Rosner BA. Nonparametric methods. In: Fundamentals of biostatistics. Boston: Duxbury Press, 1982:277-81.

25 Sokal RR, Rohlf FJ. Assumption of analysis of variance. In Biometry. New York: WH Freeman, 1995:427-31.

26 Counter CM, Gupta J, Harley CB, et al. Telomerase activity in normal leukocytes and in hematologic malignancies. Blood 1995;85:2315-30.

27 Onda K, Davis RL, Wilson CB, et al. Regional differences in bromodeoxyuridine uptake, expression of $\mathrm{Ki}-67$ protein and nucleolar organizer region counts in glioblastoma multiforme. Acta Neuropathol 1994;87:586-93.
28 Shroyer KR, Stephens JK, Silverberg SG, et al. Telomerase expression in normal endometrium, endometrial hyperplasia, and endometrial adenocarcinoma. Int f Gynecol Pathol 1997;16:225-32.

29 Kyo S, Takakura M, Kohama T, et al. Telomerase activity in human endometrium. Cancer Res 1997;57:610-14.

30 Landberg G, Nielsen NH, Nilsson P, et al. Telomerase activity is associated with cell cycle deregulation in human breast cancer. Cancer Res 1997;57:549-54.

31 Sallinen P, Miettinen H, Sallinen S-L, et al. Increased expression of telomerase RNA component is associated with increased cell proliferation in human astrocytomas. Am F Pathol 1997;150:1159-64.

32 Sidransky B, Mikkelsen T, Schwechheimer K, et al. Clonal expansion of p53 mutant cells is associated with brain tumor progression. Nature 1992;355:846-7.

33 Louis, DN. A molecular genetic model of astrocytoma histopathology. Brain Pathol 1997;7:755-64.

34 Burger PC, Rawlings CE, Cox EB, et al. Clinicopathologic correlations in oligodendroglioma. Cancer 1987;59. 1345-52.

35 Mork SJ, Halvorsen TB, Lindegaard K-F, et al. Oligodendroglioma. Histologic evaluation and prognosis. $\mathscr{f} \mathrm{Neu}$ ropathol Exp Neurol 1986;45:65-78.

36 Nelson JS, Petito CK, Scott CB, et al. High cell density and nuclear pleomorphism do not predict shortened survival in oligodendroglial tumors: report from radiation therapy oncology group (RTOG study 830). I Neuropathol Exp Neurol 1996;55:638A.

37 Schiffer D, Dutto A, Cavalia P, et al. Oligodendrogliomas: prognostic factors and proliferation markers. $\mathcal{F}$ Neuropathol Exp Neurol 1996;55:639A.

38 Coons SW, Johnson PC. Mib-1/Ki-67 labeling index predicts patient survival for oligodendroglial tumors. $\mathcal{F}$ Neuropathol Exp Neurol 1995;54:440A.

39 Kros JM, Hop WC, Godschalk JJ, et al. Prognostic value of the proliferation-related antigen $\mathrm{Ki}-67$ in oligodendrogliomas. Cancer 1996;78:1107-13. 